2. Anwendung einer extremen Belastung an den gleichen Tieren. Diese Belastung sollte sowohl den Produktionsbedingungen entsprechen als auch die Grenze der Belastbarkeit der Tiere erreichen.

3. Nach Differenzierung der Tiere aufgrund der Überlebensrate in solche mit ausreichender und nicht ausreichender Adaptationsfähigkeit, muss anhand der Reaktion der gleichen Tiere unter mässiger Belastung festgestellt werden, ob eine hinreichend genaue Differenzierung in adaptationsfähige und nicht adaptatationsfähige Individuen aufgrund der ermittelten physiologischen Parameter möglich ist.

4. Durchführung einer standardisierten mässigen Belastung an einem grösseren Versuchstierbestand zur Ermittlung von Parametern als Basis für eine Selektion.

\title{
KONSTITUTIONSKRITERIEN BEI SCHWEINEN UNTERSCHIEDLICHER NUTZUNGSRICHTUNG UND GRÖSSE
}

J. UnShelm, H. Hohns, B. Oldigs und B. RühL. - Max-Planck-Institut für Tierzucht und Tievernährung, Arbeitsgruppe Konstitutionsforschung, Trenthorst über Bad Oldeslex (B.R. D.). - Institut für Tierzucht und Haustiergenetik der Universität Göttingen (B. R. D.). - Institut für Veterinärpathologie der Freien Universität Berlin West.

Bei Schweinen unterschiedlicher Nutzungsrichtung und Grösse (Deutsche Landrasse, Piétrain, Deutsches Weideschwein, Mangalica, Göttinger Miniaturschwein) wurden in Abhängigkeit vom Lebensalter vergleichende Untersuchungen über die Mast- und Schlachtleistung einschliesslich der Fleischbeschaffenheit sowie über die Morphologie und Physiologie wichtiger Organsysteme durchgeführt. Die Ergebnisse lassen erkennen, dass auch Schweine, die zu Rassen mit einem sehr geringen Muskelbildungsvermögen gehören, morphologische und physiologische Voraussetzungen aufweisen, die ungünstiger als die anderer Tierarten sind und die bisher zum Teil mit der einseitigen Selektion der Fleischschweine erklärt wurden. Andererseits konnte festgestellt werden, dass bei den Fleischschweinrassen und hier besonders bei den Piétrainschweinen hinsichtlich nahezu aller geprüften Organsysteme mit Ausnahme der Aktivität des Wachstumshormons Minderleistungen gegenüber den Fettsch weinrassen vorliegen oder zu erwarten sind. Aus den Ergebnissen wird gefolgert, dass die meist nicht sehr günstigen anatomischen und physiologischen Voraussetzungen beim Schwein durch die Selektion auf ein höheres Muskelbildungsvermögen noch weiter verschlechtert wurden. Es wird vorgeschlagen, mehrere physiologische Parameter zunächts versuchsweise und dann routinemässig im Rahmen der Mastleistungsprüfungen zu erfassen. Gleichzeitig könnte dabei der Erblichkeitsantiel von Konstitutionsmerkmalen geschätzt werden. Das wäre eine wichtige Voraussetzung für eine gezielte züchterische Beeinflussung der Konstitution und damit der Widerstandsfähigkeit des Schweines.

\section{RELATIONS ENTRE ILES RÉACTIONS DU PORC A UN CHOC THERMIQUE ET LA QUALITÉ DE LA VIANDE}

J. Charpentier, G. Monin et L. Ollivier*. - Station de Recherches sur la viande, 63 - Theix. -_. * Station de Génétique quantitative et appliquée, I. N. R. A., 78-Jouy-en-Josas (France).

269 porcs Large White (LW $\times$ LW) (échantillon A) et 296 porcs (échantillon B) appartenant aux races ou croisements suivants, Piétrain $(\mathrm{P} \times \mathrm{P})$, Piétrain $\times$ Large White $(\mathrm{P} \times \mathrm{LW})$, Porc Blanc de l'Ouest $\times$ Piétrain $(\mathrm{PBO} \times \mathrm{P})$ et Porc Blanc de l'Ouest $\times$ Large White $(\mathrm{PBO} \times \mathrm{LW})$, ont été soumis à une température de $4^{\circ} \mathrm{C}$ pendant vingt minutes, une semaine avant leur abattage, à environ Ioo $\mathrm{kg}$ de poids vif. La température musculaire, le rythme respiratoire et le rythme cardiaque ont été enregistrés au début et à la fin du traitement thermique. Des mesures de couleur, de rétention d'eau et de $\mathrm{pH}$ ont été prises sur le jambon, 24 heures après l'abattage. La distance entre l'élevage et l'abattoir était de $30 \mathrm{~km}$ pour A et $90 \mathrm{~km}$ pour B. Les animaux disposaient de I 2 à I 8 heures de repos entre leur arrivée à l'abattoir et l'abattage. L'expérience s'est déroulée entre août et novembre I969 pour A et mai et décembre I970 pour B. 
Les corrélations entre les mesures sur l'animal vivant et les caractéristiques de la viande ont été estimées intra-groupe de porcs abattus le même jour. Ces corrélations sont faibles et variables selon la race ou l'échantillon considéré. Aucune corrélation multiple n'est significative entre les mesures sur la viande et les mesures sur le vivant. Des différences significatives existent entre races (dans l'échantillon B) pour la température musculaire, le rythme respiratoire initial, la rétention d'eau et le $\mathrm{pH}$. Les porcs $\mathrm{P} \times \mathbf{P}$ ont les plus faibles températures musculaires, la respiration la plus lente et l'accélération la plus marquée du rythme cardiaque sous l'effet de la chaleur. La qualité de la viande est la plus basse en $\mathrm{P} \times \mathrm{LW}$.

Dans les deux échantillons, des différences significatives existent entre groupes de porcs abattus à des jours différents. Ces différences paraissent plus importantes pour les caractéristiques de la viande que pour les réactions physiologiques de l'animal vivant.

Au vu de ces résultats, il y a peu à espérer d'une sélection sur les critères physiologiques retenus dans cette étude pour améliorer la qualité de la viande chez le porc, sauf peut-être pour les races du type Piétrain. Quoi qu'il en soit, il semble nécessaire d'améliorer la précision des mesures physiologiques et de standardiser les conditions de transport et d'abattage avant de pouvoir apporter une réponse définitive à la question de la sélection du porc vivant sur la qualité de sa viande.

\title{
Physiological, asPects OF MEAT QUALITy AND ADAPTATION IN PIGS (1)
}

David Lister. - U. K. Meat Research Institute, Langford, Bristol BS18 7DY (Great Britain).

Pigs containing significantly increased amounts of lean in their carcasses tend to die more readily at times of stress. Animals surviving to the usual time of slaughter, frequently produce meat of less than optimal quality. This quality cannot be significantly improved by the same modification of slaughter and handling practices from which so-called stress resistant types of pig benefit.

Evidence is presented that mesomorphism in pigs is associated with low resting values for plasma cortisol and lower levels after appropriate adrenal stimulation when compared with less mesomorphic types of pig. Attempts to correct this condition have led to improvements in the quality of the meat when the animals are slaughtered.

A complete explanation of the aetiology of the syndrome still eludes us.

\section{Interaction génotype-alimentation}

\author{
DIE INTERAKTION ZWISCHEN HERDENNIVEAU \\ UND VATER BEI DER MILCHLEISTUNG DES FLECKVIEHS IN BAYERN
}

G. Averdunk und H. Alps. - Bayer. Landesanstalt für Tierzucht, Grub (B. R. D.).

Die Interaktion zwischen Vater und Herdenniveau (3 Stufen : $3650 \mathrm{~kg}, 3650-4 \mathrm{Ig9} \mathrm{kg}$, $4199 \mathrm{~kg}$ ) wurde an einer Stichprobe des Fleckviehs in Bayern untersucht. Das Material beschränkte sich auf Nachkommen von KB-Bullen, die in jeder Stufe wenigstens ro Töchter hatten, sodass 456 Väter mit 68707 Nachkommen berücksichtigt wurden. Die Ergebnisse zeigen, dass die Interaktionen zwar signifikant sind, aber der relative Anteil mit $0.2 \mathrm{I}, 0 . \mathbf{I} 7 \mathrm{bzw}$.

(1) This paper was prepared whilst the author was a visiting research worker at Danish Meta Research Institute, Roskilde, Denmark. 